\title{
Erratum
}

\section{Citrus fruit intake and stomach cancer risk: a quantitative systematic review}

\author{
Jong-MYon BAE ${ }^{1,2}$, Eun JA LeE ${ }^{3}$, and Gordon GuYatT ${ }^{2}$ \\ ${ }^{1}$ Department of Preventive Medicine, Cheju National University College of Medicine, No. 1 Ara-1-dong, Jeju, Jejudo 690-756, Korea \\ ${ }^{2}$ CLARITY Research Group, Department of Clinical Epidemiology \& Biostatistics, Health Sciences Centre, McMaster University, Hamilton, \\ Ontario, Canada \\ ${ }^{3}$ Department of Radiology, Myongii Hospital, Kwandong University, College of Medicine, Koyang, Korea
}

Gastric Cancer (2008) 11: 23-32

DOI 10.1007/s10120-007-0447-2

An error occurred in the first sentence of the final paragraph in the section Statistical analyses on page 24. The sentence should read as follows:

We used The Cochrane Collaboration software RevMan 4.2 to analyze the extracted data with both fixed-effect and random-effect model analysis [25].

The online version of the original article can be found at http://dx.doi.org/10.1007/s10120-007-0447-2 